\title{
Study of Polysilicon Position-Sensitive Detector (PSD)
}

\author{
Hideo Muro* \\ Department of Electrical, Electronics and Computer Engineering, Chiba Institute of Technology, \\ 2-17-1 Tsudanuma, Narashino, Chiba 275-0016, Japan
}

(Received March 31, 2008; accepted July 16, 2008)

Key words: polycrystalline silicon, microsensor, photodiode, PSD

A position-sensitive detector (PSD) fabricated using polysilicon films by a standard LSI fabrication process has been designed and its basic characteristics for sensor applications have been evaluated, together with the electrical characteristics of polysilicon photodiodes. A polysilicon PSD can be used as an optical position sensor on LSI chips without additional areas or process steps. Although the optoelectric conversion efficiency of a polysilicon PSD with a thickness of $335 \mathrm{~nm}$ irradiated with a near-IR light source was very low owing to small absorption coefficients and a short diffusion length of minority carriers in polysilicon films, a linear relationship between light position and output current was obtained. Using polysilicon films with a thickness of $1 \mu \mathrm{m}$, we improved the optoelectric conversion by about one order and obtained a hyperbolic-sine position dependence.

\section{Introduction}

Polycrystalline silicon (polysilicon) thin films deposited by LPCVD are widely used as gate materials of MOSFETs and resistors in integrated circuits. The electrical properties such as resistivity and Hall mobility of polysilicon films have been intensively studied and analyzed.(1-5) With advances in micro-electro-mechanical-systems (MEMS) technology, the applications of polysilicon thin films have been extended to silicon sensors as piezoresistive gauges ${ }^{(6-9)}$ or thermocouples ${ }^{(10-13)}$ owing to their comparatively large piezoresistive coefficients and Seebeck coefficients, in addition to their structure made on a silicon oxide layer for high-temperature operation or thermal isolation.

As a photosensing device, a polysilicon thin film is, in principle, unsuitable owing to the small absorption of near-infrared light and short carrier lifetime in the film, which result in small optoelectric conversion efficiencies. If, however, it is integrated onto an integrated-circuit chip without any additional cost, it can be a potential candidate for photodetectors in some applications.

*Corresponding author: e-mail: hideo.muro@it-chiba.ac.jp 
Among various optical sensing systems, optical linear position sensors can be easily constructed by forming a pn junction and utilizing one side as a distributed resistor with electrodes on both sides, which is called a position-sensitive detector (PSD). The ratio of a pair of output currents from the electrodes depends on the position of light irradiated on the PSD, and by signal processing of the output currents the position can be detected in one or two dimensions.(14-19) If PSDs are formed using polysilicon thin films, position sensors can be easily realized by a standard LSI process without any additional fabrication steps and connected with signal processing circuitry on the chip, even though their performances are not better than those of the conventional PSDs.

In this study, the basic characteristics of pn junctions laterally formed in polysilicon films using a standard LSI fabrication process were investigated and a PSD using polysilicon films was designed, fabricated, and tested as an optical position sensor.

\section{Materials and Methods}

\subsection{Test samples}

Test devices for evaluating the sensing properties of polysilicon films were designed, together with their mask patterns. The masks included diodes for evaluating the basic characteristics of polysilicon pn junctions and a test device of a polysilicon PSD. Two types of diode, (a) $\mathrm{p}^{+} / \mathrm{n} / \mathrm{n}^{+}$-type and (b) $\mathrm{p}^{+} / \mathrm{p} / \mathrm{n}^{+}$-type, were considered, where $5 \mu \mathrm{m}$ lightly doped background regions of n-type or p-type were sandwiched between the $\mathrm{p}^{+}$and $\mathrm{n}^{+}$ regions to reduce leakage current, as shown in Fig. 1. The PSD test device consisted of a p-type resistor stripe, $\mathrm{p}^{+}$comblike electrodes connected to both sides of the stripe perpendicularly, and $\mathrm{n}^{+}$comblike electrodes facing the $\mathrm{p}^{+}$electrodes. The entire device layout is shown in Fig. 2.

The test samples were fabricated using a conventional IC process. The starting material was a 5 inch, p-type silicon wafer. After forming a 580 -nm-thick initial oxide layer, various diffusion layers were formed consecutively to integrate transistors into the chip. Next, a 350-nm-thick polysilicon layer was deposited by LPCVD at $625^{\circ} \mathrm{C}$ and doped selectively by ion implantation using a photoresist as a mask. Samples with

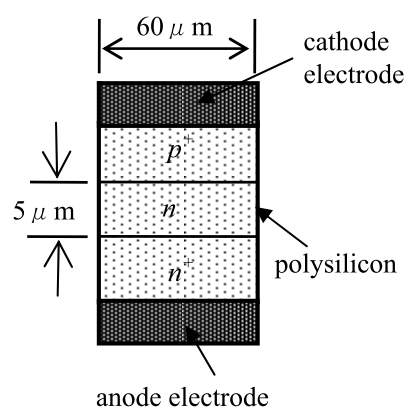

(a)

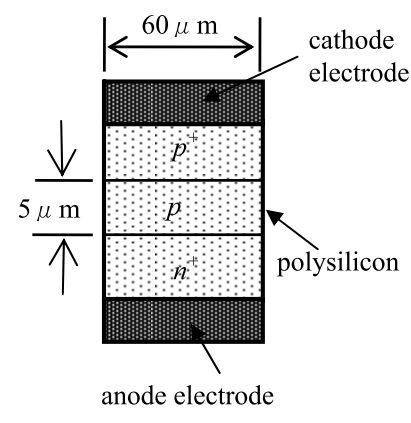

(b)

Fig. 1. Schematic drawings of (a) $\mathrm{p}^{+} / \mathrm{n} / \mathrm{n}^{+}$-type and (b) $\mathrm{p}^{+} / \mathrm{p} / \mathrm{n}^{+}$-type polysilicon diodes. 


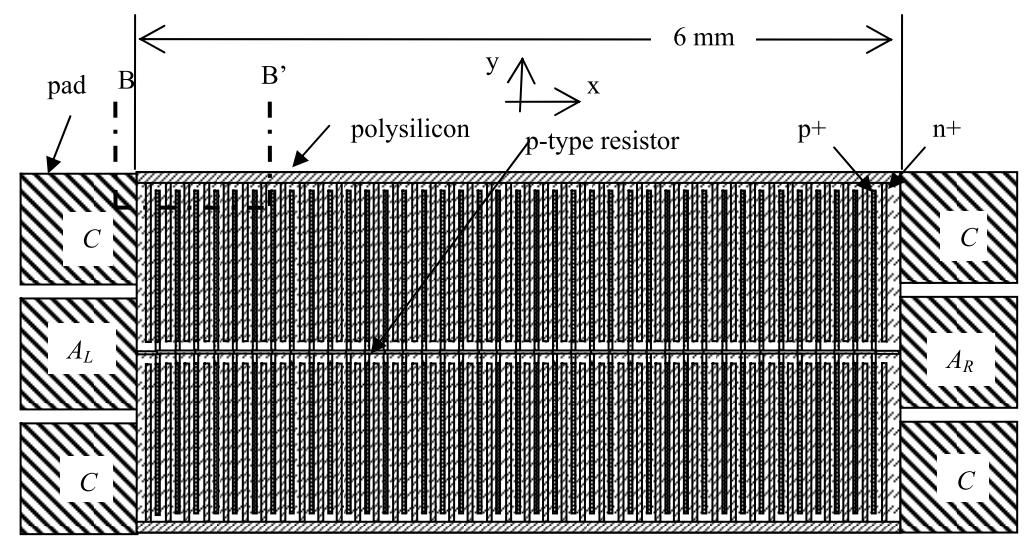

pad

(a)

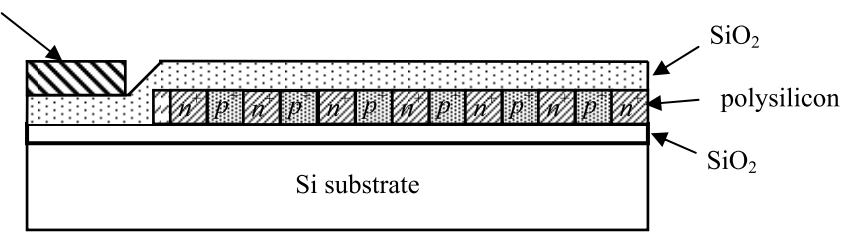

(b)

Fig. 2. (a) Schematic view of designed polysilicon PSD and (b) its B-B' cross section.

thicker polysilicon films $(1 \mu \mathrm{m})$ were also fabricated. Phosphorus and boron were implanted to form the n-type and p-type regions, respectively, at doses ranging from 5 $\times 10^{14} / \mathrm{cm}^{2}$ to $5 \times 10^{15} / \mathrm{cm}^{2}$. The $\mathrm{p}^{+}$and $\mathrm{n}^{+}$regions were implanted at a dose of $1 \times 10^{16} / \mathrm{cm}^{2}$. The resistor stripes of the PSD were implanted at a B dose of $5 \times 10^{15} / \mathrm{cm}^{2}$. The implant energies were $100 \mathrm{keV}$ and $40 \mathrm{keV}$ for phosphorus and boron, respectively. After patterning the polysilicon layer by dry etching, it was thermally oxidized to form a 100 -nm-thick buffer oxide layer and annealed for $30 \mathrm{~min}$ at $900^{\circ} \mathrm{C}$ to activate the implanted dopants. A 700-nm-thick silicon oxide layer was deposited by CVD and contact holes were etched. Finally, a 1- $\mu \mathrm{m}$-thick aluminum layer was deposited and patterned to form interconnections, and sintered in $\mathrm{H}_{2}$.

\subsection{Measurements}

The fabricated test chips of the polysilicon diodes with various doses were diced and mounted on ceramic packages with epoxy glue. The forward and reverse I-V characteristics were measured together with their temperature dependences. The test chips of polysilicon PSD were also prepared and evaluated using the experimental setup shown in Fig. 3. The width $W$ and the length $L$ of the PSD are 6 and $1.2 \mathrm{~mm}$, respectively, and the grain size was estimated to be several tens of $\mathrm{nm}$. The electrodes $A_{\mathrm{R}}$ and $A_{\mathrm{L}}$ connected to both ends of the p-type resistor stripe in the PSD are biased by 


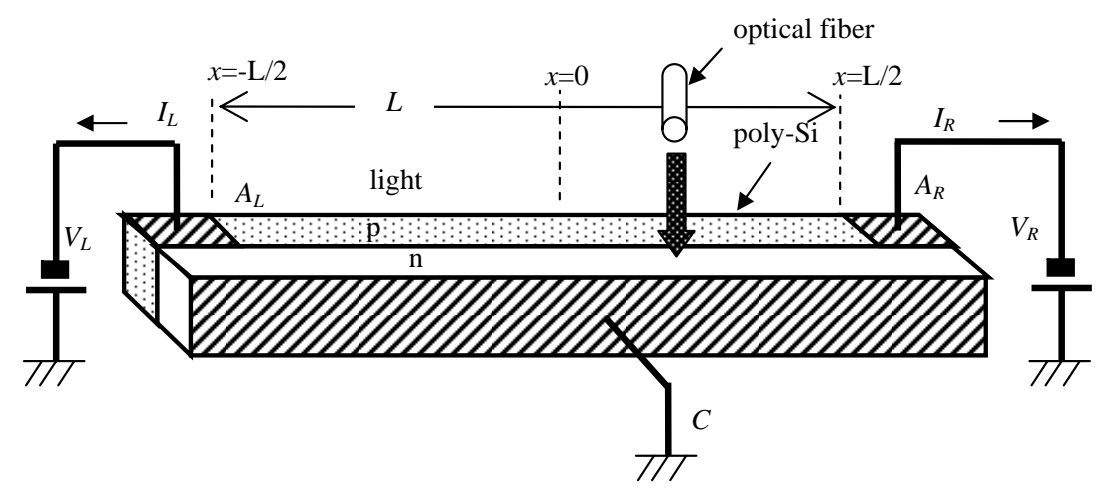

Fig. 3. Experimental setup for measuring output current vs position characteristics of polysilicon PSD.

constant voltage sources, $V_{\mathrm{R}}$ and $V_{\mathrm{L}}$. A portion of the PSD is irradiated with a near-IR light source $(\lambda=780 \mathrm{~nm})$ through an optical fiber. The optical power from the optical fiber is about $1 \mathrm{~mW}$. The spot size of the light was about $1 \mathrm{~mm}$. The output current of the PSD was measured while the position of the light, $x$, was scanned from one end to the other.

\section{Results and Discussion}

\subsection{Polysilicon diode}

Figure 4 shows the temperature dependence of the forward I-V characteristics of the $\mathrm{p}^{+} / \mathrm{n} / \mathrm{n}^{+}$-type polysilicon diode with a $\mathrm{P}^{+}$dose of $5 \times 10^{14} \mathrm{~cm}^{-2}$. The characteristics of all samples resemble those of the single crystal silicon diode and can be approximated, in the medium current range up to the high-level injection region, as follows:

$$
I=I_{\mathrm{s}}\left(\exp \frac{q V}{n k T}-1\right)
$$

where $I, V_{\mathrm{F}}, I_{\mathrm{s}}, n, k$, and $T$ are the anode current, the applied forward bias voltage, the saturation current of the pn junction, the ideality factor, the Boltzmann constant, and the absolute temperature, respectively. The current increases with temperature. The temperature dependences of the saturation current $I_{s}$, which were calculated using Eq. (1) and a fitting parameter $n=1.4$, are plotted as a function of $1 / k T$ in Figs. 5 and 6, for the $\mathrm{p}^{+} / \mathrm{n} / \mathrm{n}^{+}$-type diode and $\mathrm{p}^{+} / \mathrm{p} / \mathrm{n}^{+}$-type diode, respectively. The saturation current $I_{\mathrm{s}}$ increases with background dose for both the $\mathrm{p}^{+} / \mathrm{n} / \mathrm{n}^{+}$-type diode and $\mathrm{p}^{+} / \mathrm{p} / \mathrm{n}^{+}$-type diode. From these figures, the temperature dependence of the saturation current $I_{\mathrm{s}}$ can be expressed as a form of $\exp \left(-E_{\mathrm{a}} / \mathrm{kT}\right)$, where $E_{\mathrm{a}}=0.6-0.7 \mathrm{eV}$. This finding indicates that $I_{\mathrm{s}}$ is proportional to the intrinsic carrier concentration, $n_{\mathrm{i}}$, rather than $n_{\mathrm{i}}^{2}$; therefore the recombination in the 


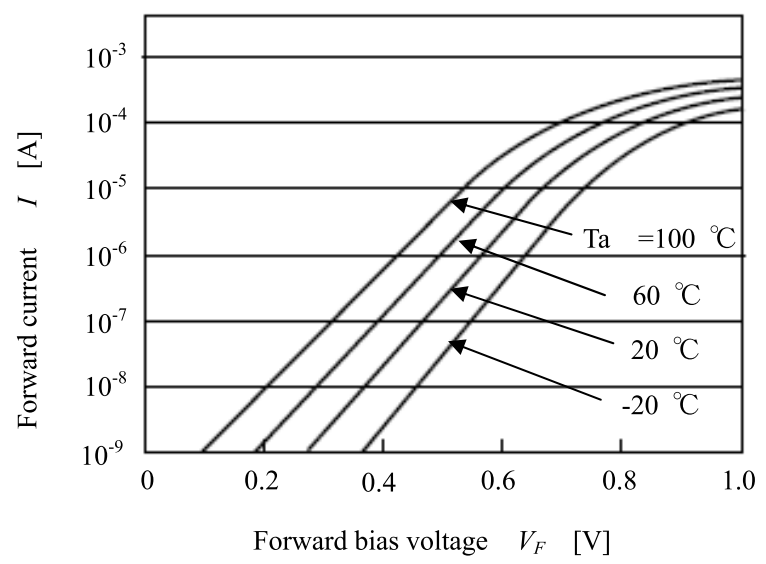

Fig. 4. Measured temperature dependence of forward I-V characteristics of $\mathrm{p}^{+} / \mathrm{n} / \mathrm{n}^{+}$-type polysilicon diode with $\mathrm{P}$ dose of $5 \times 10^{14} \mathrm{~cm}^{-2}$.

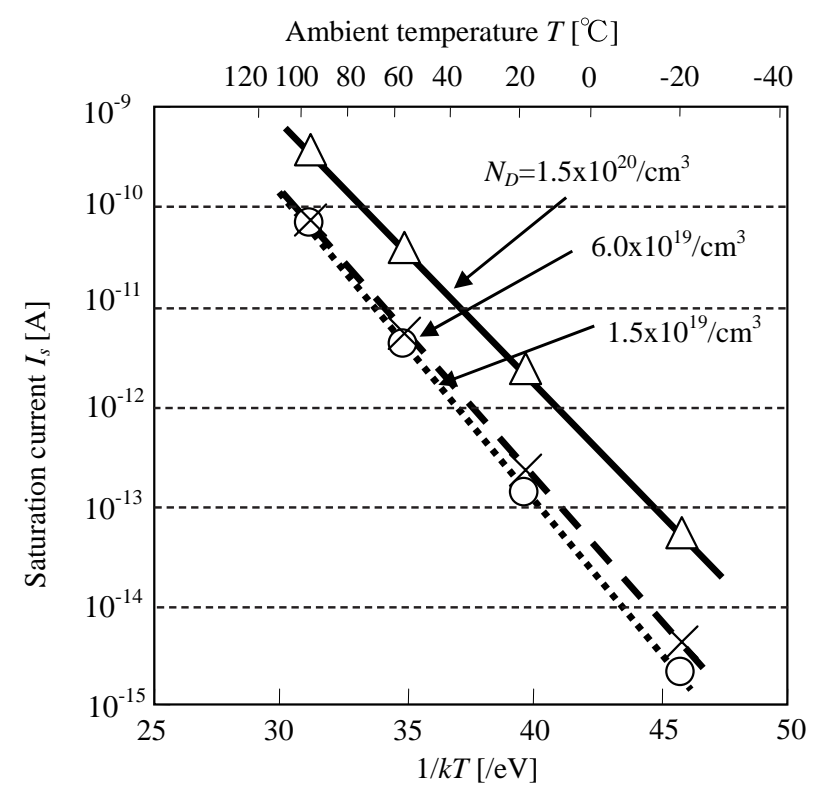

Fig. 5. Temperature dependence of saturation current $I_{\mathrm{s}}$ of $\mathrm{p}^{+} / \mathrm{n} / \mathrm{n}^{+}$-type polysilicon diodes with various phosphorus doses. 


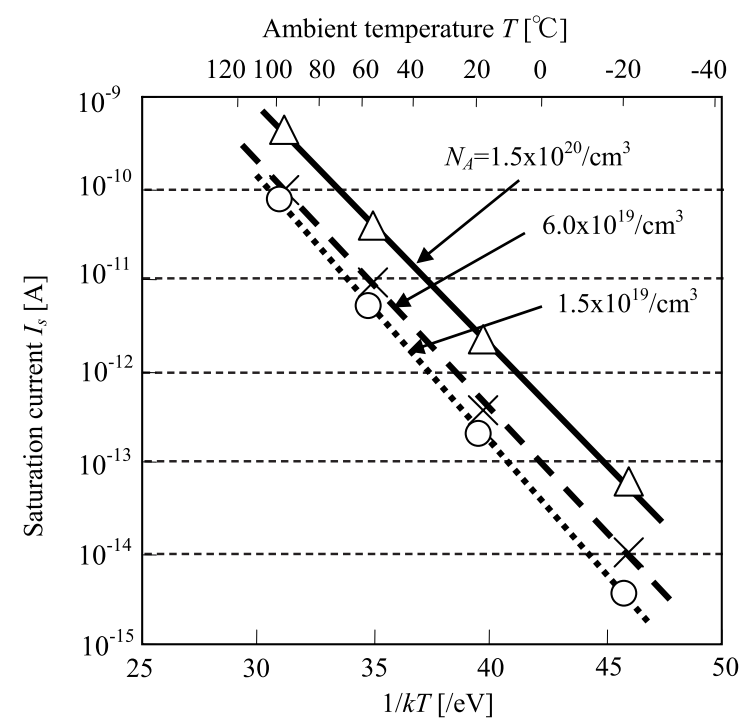

Fig. 6. Temperature dependence of saturation current $I_{\mathrm{s}}$ of $\mathrm{p}^{+} / \mathrm{p} / \mathrm{n}^{+}$-type polysilicon diodes with various boron doses.

depletion region of the pn junction predominantly contributes to $I_{\mathrm{s}}$.

The measured temperature dependence of reverse I-V characteristics of the $\mathrm{p}^{+} / \mathrm{p} / \mathrm{n}^{+}-$ type polysilicon diode with a B dose of $5 \times 10^{14} \mathrm{~cm}^{-2}$ is shown in Fig. 7. The leakage current of the diode, $I_{\text {leak }}$, increases with the reverse bias voltage $V_{\mathrm{R}}$ up to a breakdown voltage of about $6 \mathrm{~V}$, at which reverse current increases markedly. Leakage current also increases gradually with temperature, although breakdown voltage is independent of temperature. The activation energies of the leakage current $E_{\mathrm{a}}$ calculated from the assumed dependence of $\exp \left(-E_{\mathrm{a}} / \mathrm{kT}\right)$ range from 0.06 to $0.15 \mathrm{eV}$ for the $\mathrm{p}^{+} / \mathrm{n} / \mathrm{n}^{+}$-type diode, and from 0.05 to $0.1 \mathrm{eV}$ for the $\mathrm{p}^{+} / \mathrm{p} / \mathrm{n}^{+}$-type diode.

\subsection{Polysilicon PSD}

Using the fabricated test samples of polysilicon PSD, the photosensitivity $S$ was measured. Photosensitivity is defined as a ratio of the total photocurrent, $I_{0}=I_{\mathrm{R}}+I_{\mathrm{L}}$, to the input optical power $P_{0}$, that is,

$$
S=\frac{I_{0}}{P_{0}}
$$

Figure 8 shows the measured results for PSD samples of various background doping concentrations, biased at a constant voltage $V_{\mathrm{R}}=V_{\mathrm{L}}=0 \mathrm{~V}$. Photosensitivity increases as background doping concentration decreases at which the width of the depletion region and the diffusion length of the minority carrier are larger. For a polysilicon film thickness $t$ of $1 \mu \mathrm{m}$, the photosensitivity $S$ is fourfold that of standard samples or higher. 


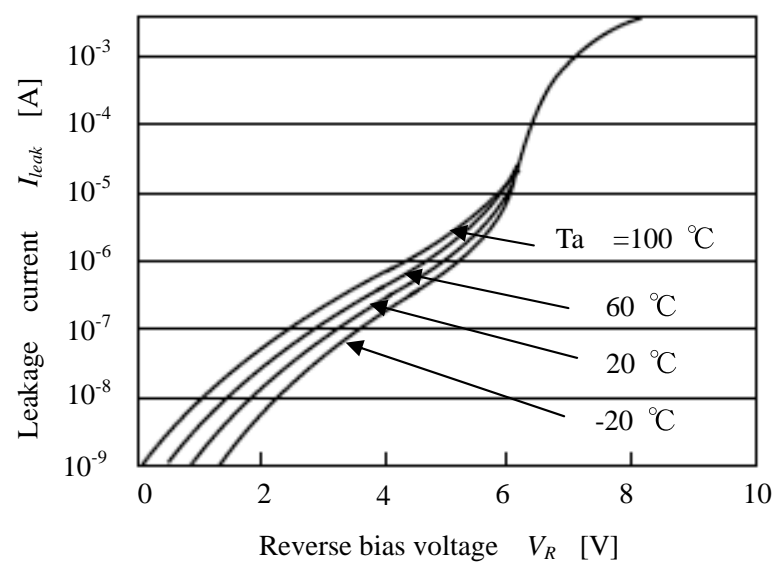

Fig. 7. Measured temperature dependence of reverse $I-V$ characteristics of $\mathrm{p}^{+} / \mathrm{p} / \mathrm{n}^{+}$-type polysilicon diode with $\mathrm{B}$ dose of $5 \times 10^{14} \mathrm{~cm}^{-2}$.

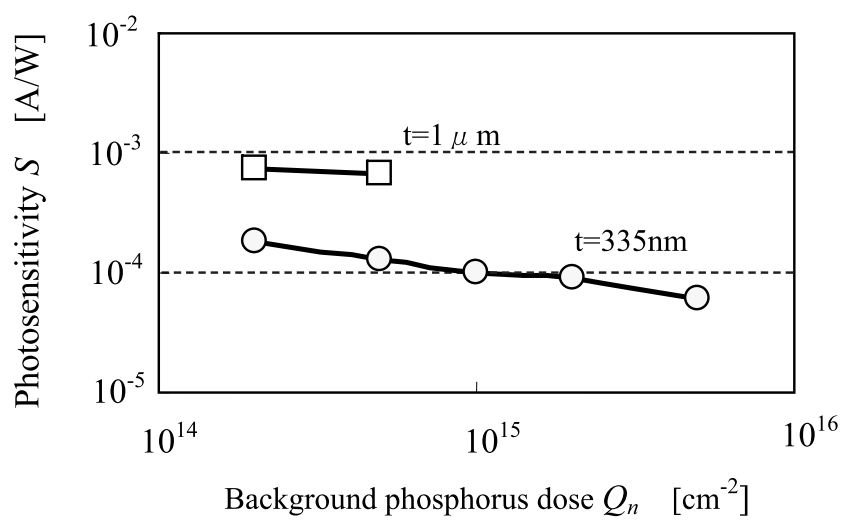

Fig. 8. Dependences of photosensitivity of fabricated polysilicon PSD on background phosphorus dose with bias voltage $V_{\mathrm{R}}=V_{\mathrm{L}}=0 \mathrm{~V}$.

The conversion efficiency $\eta$ can be defined as follows:

$$
\eta=\frac{h \nu \times I_{0}}{q \times P_{0}}
$$

where $h$ and $v$ are the Plank constant and the optical frequency, respectively. The conversion efficiency $\eta$ of the 335-nm-thick polysilicon PSD was less than $0.3 \%$, 
while that of the $1-\mu \mathrm{m}$-thick polysilicon PSD was greater than $1 \%$. Figure 9 shows the measured photosensitivities similar to those shown in Fig. 8 for a bias voltage $V_{\mathrm{R}}=V_{\mathrm{L}}$ $=2 \mathrm{~V}$. In this case, leakage current cannot be neglected and photocurrent was obtained by subtracting leakage current from the measured output current. The photosensitivity becomes larger than that for $0 \mathrm{~V}$ bias owing to the widening of the depletion region, particularly for low-doping samples. However, for PSD application, the leakage current causes a nonlinearity, which deteriorates the accuracy of position-sensing characteristics. The position-sensing characteristics were evaluated out with $V_{\mathrm{R}}=V_{\mathrm{L}}=0 \mathrm{~V}$ to avoid this nonlinearity.

The output current of the PSD was measured by scanning the light position $x$ using the manipulator. Figure 10 shows the output currents of the PSD, $I_{R}$ and $I_{L}$, normalized to the total output current $I_{0}=I_{\mathrm{R}}+I_{\mathrm{L}}$, vs the light position $x$ for the sample with a background dose of $2 \times 10^{14} / \mathrm{cm}^{2}$ and a film thickness of $335 \mathrm{~nm}$. The measured values of $I_{\mathrm{R}}$ and $I_{\mathrm{L}}$ are proportional to the position and their linearity is very good. Figure 11 shows similar results for the sample with the same background dose and a film thickness

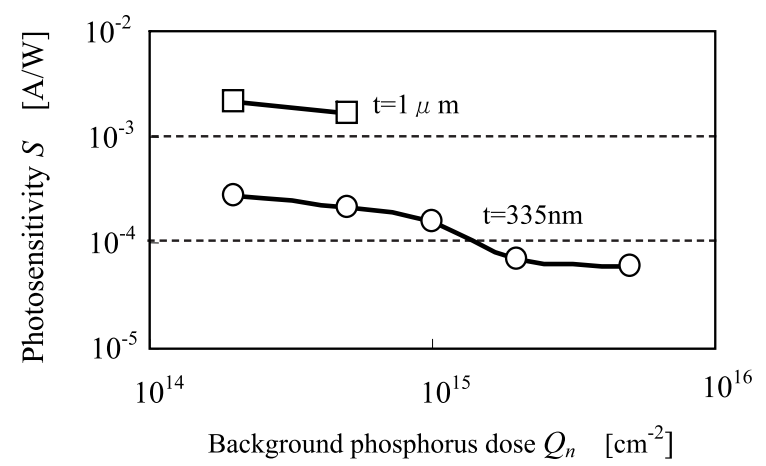

Fig. 9. Dependences of photosensitivity of fabricated polysilicon PSD on background phosphorus dose with bias voltage $V_{\mathrm{R}}=V_{\mathrm{L}}=2 \mathrm{~V}$.

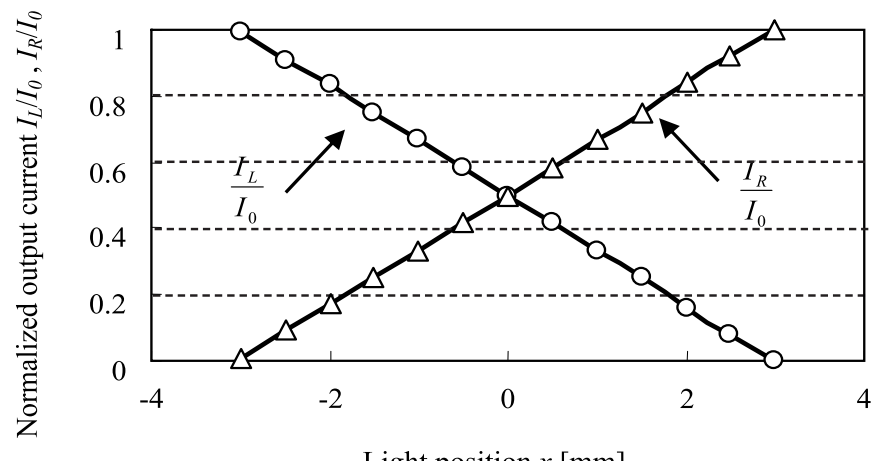

Light position $x[\mathrm{~mm}]$

Fig. 10. Light position dependences of measured output current of PSD with polysilicon thickness of $335 \mathrm{~nm}$ and background phosphorus dose of $2 \times 10^{14} \mathrm{~cm}^{-2}$. 
of $1 \mu \mathrm{m}$. In this case, the position dependence cannot be regarded to be linear and the currents saturate around both sides. The normalized output $k=\left(I_{\mathrm{R}}-I_{\mathrm{L}}\right) /\left(I_{\mathrm{R}}+I_{\mathrm{L}}\right)$ is plotted in Fig. 12 for the above-mentioned samples. The characteristics of the latter sample can be approximated by a hyperbolic-sine curve, while those of the former sample are linear. These results can be explained by the nonlinear I-V characteristics of the polysilicon resistor used in the PSD.

The electrical properties of polysilicon resistors can be described by the carriertrapping model. When the voltage across the undepleted crystallite is negligible owing to sufficient doping, the applied voltage is consumed entirely at each grain boundary junction and the I-V characteristics of a PSD with a length of $l$ can be approximately expressed as follows:(2)

$$
I=2 I_{\mathrm{p}} \sinh \left(\frac{q L_{\mathrm{g}} V}{2 k T l}\right)
$$

where

$$
I_{\mathrm{p}}=q N W t \sqrt{\frac{k T}{2 \pi m^{*}}} \exp \left(-\frac{q V_{\mathrm{B}}}{k T}\right)
$$

Here, $q, N, m^{*}, V_{\mathrm{B}}$, and $L_{\mathrm{g}}$ are the electron charge, average carrier concentration, effective mass of the carrier, potential barrier height, and grain size, respectively.

When a PSD with a length of $\mathrm{L}$ is biased with $V_{\mathrm{R}}=V_{\mathrm{L}}=0 \mathrm{~V}$ and is irradiated by a light source at $x$, the optically generated carriers cause a pair of output photocurrents,

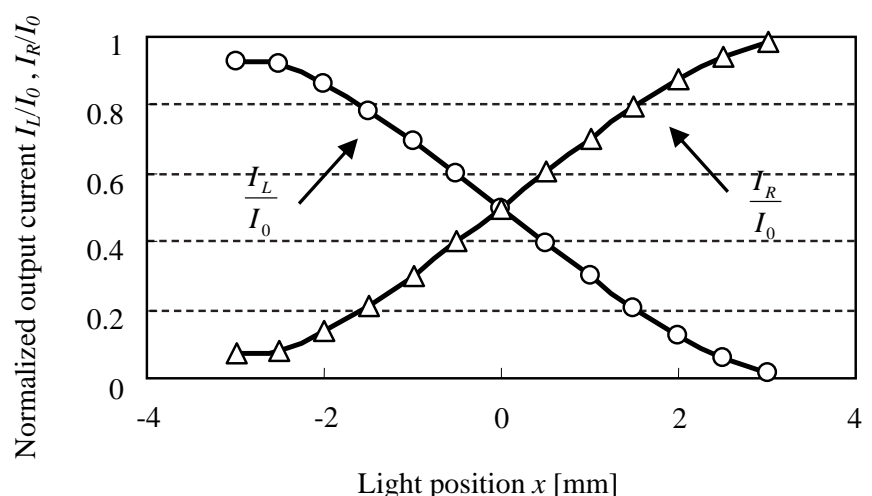

Fig. 11. Light position dependences of measured output current of PSD with polysilicon thickness of $1 \mu \mathrm{m}$ and background phosphorus dose of $2 \times 10^{14} \mathrm{~cm}^{-2}$. 


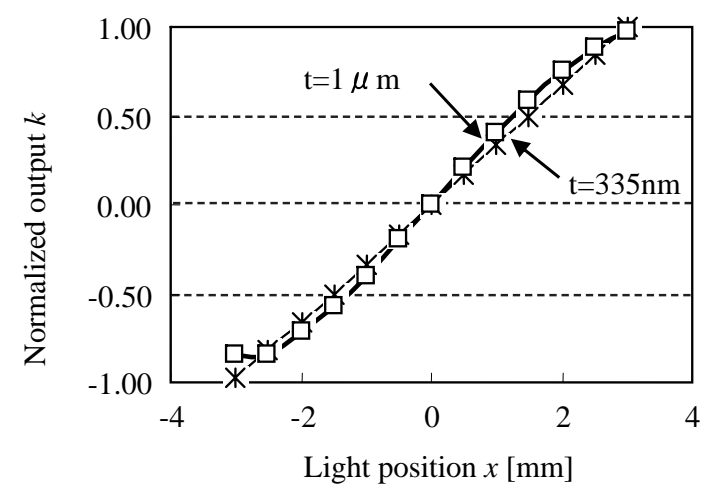

Fig. 12. Light position dependences of the normalized output of PSDs with polysilicon thicknesses $t=335 \mathrm{~nm}$ and $1 \mu \mathrm{m}$.

resulting in a voltage at $x, V_{\mathrm{ir}}$ Each of the output currents of the PSD, $I_{\mathrm{R}}$ and $I_{\mathrm{L}}$, can be expressed as a function of the light position $x$ as follows:

$$
\begin{aligned}
& I_{\mathrm{R}}=2 I_{\mathrm{P}} \sinh \left\{\frac{q L_{\mathrm{g}} V_{\text {ir }}}{2 k T(L / 2-x)}\right\}, \\
& I_{\mathrm{L}}=2 I_{\mathrm{p}} \sinh \left\{\frac{q L_{\mathrm{g}} V_{\text {ir }}}{2 k T(L / 2+x)}\right\} .
\end{aligned}
$$

These output currents have compressed dependences on the light position if the voltage drop caused by the currents, $V_{\mathrm{ir}}$, is large, while they can be approximated by linear dependences on the light position if $V_{\text {ir }}$ is small. Therefore, by adjusting doping concentration and polysilicon film thickness or the light intensity, position-sensing characteristics can be modified to be linear or to show a compressed relationship depending on the requirement of the sensor.

\section{Conclusions}

Test chips for evaluating the basic properties of polysilicon photodiodes and PSDs have been designed and fabricated using a conventional IC process. The I-V characteristics of both the $\mathrm{p}^{+} / \mathrm{n} / \mathrm{n}^{+}$-type and $\mathrm{p}^{+} / \mathrm{p} / \mathrm{n}^{+}$-type polysilicon diodes were measured together with their temperature dependences. Exponential I-V relationships similar to those of single crystal silicon were obtained. The saturation currents of the diodes increase with temperature and background doping concentration, where their temperature dependences can be expressed as a form of $\exp \left(-E_{\mathrm{a}} / k T\right)$ with the activation energy $E_{\mathrm{a}}=0.6-0.7 \mathrm{eV}$. The leakage currents increase with the reverse bias voltage, $V_{\mathrm{R}}$, up to a breakdown voltage of about $6 \mathrm{~V}$, at which reverse current increases markedly. 
The temperature dependence of leakage currents was, for example, $+20,000 \mathrm{ppm} / \mathrm{K}$ at $V_{\mathrm{R}}$ $=2 \mathrm{~V}$.

The measured photosensitivity of the 335-nm-thick polysilicon PSD with the bias voltage $V_{\mathrm{R}}=V_{\mathrm{L}}=0 \mathrm{~V}$ and a light source of $\lambda=780 \mathrm{~nm}$ was approximately $10^{-4}$ $\mathrm{A} / \mathrm{W}$, which decreases gradually with increasing background doping concentration. The photosensitivity increases with the bias voltage $V_{\mathrm{R}}=V_{\mathrm{L}}=2 \mathrm{~V}$ at the expense of leakage current without irradiation that may cause nonlinear characteristics. A linear relationship between the light position and the output current ratio was obtained at the bias voltage $V_{\mathrm{R}}=V_{\mathrm{L}}=0 \mathrm{~V}$. Using polysilicon films with a thickness of $1 \mu \mathrm{m}$, we improved the photosensitivity by about one order and obtained a hyperbolic-sine position dependence, which can be applied to compressed position sensing.

\section{Acknowledgement}

The authors would like to thank members of the Nissan Research Center for help in the fabrication of the devices.

\section{References}

1 J. Y. W. Seto: J. Appl. Phys. 46 (1975) 5247.

2 N. C. Lu, L. Gerzberg, C. Lu and J. D. Meindl: IEEE Trans. Electr. Dev. 28 (1981) 818.

3 T. I. Kamins: J. Appl. Phys. 42 (1971) 4357.

4 M. E. Cowher and T. O. Sedgwick: J. Electrochem. Soc. 119 (1972) 1565

5 H. Muro, T. Mitamura and S. Kiyota: Sens. Mater. 18 (2006) 433

6 V. Mosser, J. Suski, J. Goss and E. Obermeier: Sens. Actuators, A 28 (1991) 113.

7 H. Schäfer, V. Graeger and R. Kobs: Sens. Actuators 17 (1989) 521.

8 H. Guckel and D. W. Burns: Transducers'85, 3rd Int. Conf. Solid-State Sensors and Actuators, Philadelphia (1985) 182.

9 S. Sugiyama, T. Suzuki, K. Kawahata, M. Takigawa and I. Igarashi: Proc. 6th Sensor Symp. (1986) 23.

10 G. R. Lahiji and K. D. Wise: IEEE Trans. Electr. Dev. 29 (1982) 14.

11 P. M. Sarro, A. W. Herwaarden and W. van der Vlist: Sens. Actuators, A 41,42 (1994) 666.

12 R. Lenggenhager, H. Baltes, J. Peer and M. Forster: IEEE Electr. Dev. Lett., 13 (1992) 454.

13 E. Socher, O. B.-Degani and Y. Nemirovsky: J. Microelectromech. Syst. 9 (2000) 38.

14 K. J. Erb: Proc. IEDM'85 (1985) 57.

15 W. Wang and I. J. Busch-Vishniac: IEEE Trans. Electr. Dev. 36 (1989) 2475.

16 A. K. Dutta and Y. Hatanaka: IEEE Trans. Electr. Dev. 38 (1991) $498 .$.

17 H. Muro and P. J. French: Sens. Actuators, A 21-23 (1990) 544.

18 A. Toneva and D. Sueva: Sens. Actuators 73 (1999) 210.

19 A. Mäkynen, T. Ruotsalainen, T. Rahkonen and J. Kostamovaara: Sens. Actuators, A 105 (2003) 261. 